\title{
Abundância, riqueza e diversidade de espécies de Eiphosoma Cresson 1865 (Hymenoptera, Ichneumonidae, Cremastinae) associada a hortas orgânicas
}

\author{
Abundance, richness and diversity of Eiphosoma Cresson 1865 \\ (Hymenoptera, Ichneumonidae) species associated with organic crops
}

Helena Carolina Onody ${ }^{1}$, Ivy Frizo de Melo ${ }^{1}$, Angélica Maria Penteado-Dias ${ }^{1}$

\begin{abstract}
RESUMO
O objetivo deste trabalho foi caracterizar a fauna de Eiphosoma Cresson, 1865 (Hymenoptera, Ichneumonidae, Cremastinae) em áreas de agricultura orgânica, em relação à abundância, riqueza e diversidade de espécies. Foram identificadas quatorze espécies de Eiphosoma, das quais duas são novos registros para o Brasil e três são novas espécies. A horta com intensidade mediana de manejo e diversidade vegetal apresentou os maiores valores de abundância, riqueza e diversidade em relação às hortas com maior e menor intensidade de manejo. As variáveis climáticas demonstraram exercer menor influência na fauna de Eiphosoma do que as características (composição e manejo) das hortas.
\end{abstract}

Palavras-chaves: diversidade, manejo integrado de pragas, olericultura, região neotropical, parasitoides.

\section{ABSTRACT}

The aim of this study was to characterize the Eiphosoma Cresson, 1865 (Hymenoptera, Ichneumonidae, Cremastinae) fauna in areas of organic crops production, in relation to abundance, richness and diversity of species. Fourteen Eiphosoma species were identified, two new records to Brazil and three are new species identified. The system with median intensity management showed the highest values of abundance, richness and diversity in relation with higher and lower intensity of management. The climate variables showed less influence on the Eiphosoma fauna than the characteristics (composition and management) of the systems.

Key words: diversity, integrated pest management, neotropical region, olericulture, parasitoids.

\section{Introdução}

Os parasitoides constituem importantes agentes no controle das populações de pragas agrícolas, seja pela su ocorrência natural no ambiente ou por sua utilização em programas de controle biológico. No entanto, para que haja sucesso no manejo de pragas e no desenvolvimento de estratégias de manejo local dos inimigos naturais, são imprescindíveis estudos sobre a diversidade de organismos associados aos sistemas agrícolas.

A complexidade relativa das comunidades de inimigos naturais associada aos sistemas de cultivos pode ser determinada por diferentes fatores. As propriedades rurais possuem características únicas advindas de fatores biológicos e ambientais locais, relações econômicas, estrutura social e história. Além disso, os agroecossistemas são dinâmicos e estão sujeitos a diferentes níveis de manejo, de modo que os arranjos dos cultivos e das vegetações espontâneas mudam continuamente no tempo e no espaço (Altieri, 2003; Chay-Hernandez, et al., 2006).

A biodiversidade dos agroecossistemas é essencial na estabilização da dinâmica populacional de insetos fitófagos e de seus inimigos naturais. A ocorrência dos agentes de controle em sistemas diversificados é geralmente maior devido à presença de presas e hospedeiros alternativos e à maior variedade de microhabitats (Altieri, 1994; Landis et al., 2000; Wyss \& Pfiffner, 2006).

Eiphosoma Cresson, 1865 (Hymenoptera, Ichneumonidae, Cremastinae) é um gênero do Novo Mundo, com grande riqueza de espécies na América do Sul. Compreende cerca de 50 espécies descritas, subdivididas em 9 grupos de espécies (Gauld, 2000).

1 Universidade Federal de São Carlos. Departamento de Ecologia e Biologia Evolutiva. Rodovia Washington Luis, KM 235. São Carlos - São Paulo, Brasil. helenaonody@yahoo.com.br, ivyfrizo@yahoo.com.br, angelica@ufscar.br 
Para o Brasil são citadas atualmente 17 espécies (Gauld, 2000), pertencentes aos grupos de espécies: aztecum, dentator, nigrovittatum e viticole.

As espécies deste gênero são parasitoides abundantes em ambientes abertos e degradados, incluindo agroecossistemas, onde parasitam importantes pragas agrícolas, especialmente larvas de Lepidoptera (Gauld, 2000; Fernández-Triana \& Ravelo, 2007). Apesar de sua abundância e importância, não existem trabalhos específicos sobre o grupo em hortas orgânicas, ou até mesmo outros agroecossistemas.

O presente trabalho tem por objetivos caracterizar a fauna de Eiphosoma em três áreas hortícolas orgânicas e verificar a influência de diferentes tipos de manejo e de variáveis climáticas em sua abundância, riqueza e diversidade de espécies.

\section{Material e Métodos}

As coletas foram realizadas em três áreas de agricultura orgânica:

\section{- Horta São Paulo (HSP)}

Propriedade familiar localizada dentro de um condomínio de chácaras, em Araraquara, SP $\left(21^{\circ} 42,4^{\prime} \mathrm{S}-48^{\circ} 18,8^{\prime} \mathrm{W}\right)$. A horta possui cerca de 465 $\mathrm{m}^{2}$ e utiliza como principal método de controle de pragas e de plantas invasoras o manejo mecânico, sendo algumas vezes, aplicadas caldas de fumo e de pimenta do reino. A rotatividade de cultivos é baixa e a vegetação infestante é constantemente retirada.

\section{- Horta Oyafuso (HO)}

Propriedade certificada pelo IBD (Instituto Biodinâmico), também localizada em um condomínio de chácaras em Araraquara, SP $\left(21^{\circ} 44,5^{\prime} \mathrm{S}-48^{\circ} 13,04^{\prime} \mathrm{W}\right)$. Uma área com cerca de $4.435 \mathrm{~m}^{2}$ é destinada à plantação de olerícolas. $\mathrm{O}$ principal método de controle de pragas e plantas invasoras é o mecânico, sendo também utilizado o cultural. Existe rotatividade de cultivos mais intensa do que na horta anteriormente citada, e a vegetação infestante é moderadamente conservada.

\section{- Horta da Prefeitura de São Carlos (HPSC)}

A propriedade localizada no município de São Carlos tem área de $5.320 \mathrm{~m}^{2}$ destinada à plantação de olerícolas. Esta é a propriedade que apresenta a maior rotatividade de cultivos sendo que os canteiros não são fixos e a vegetação infestante é moderadamente conservada. Possui como principal método de controle de pragas e de plantas invasoras o manejo mecânico e o cultural, além da ampla utilização de caldas e extratos vegetais.

As amostragens foram realizadas por armadilhas Malaise, instaladas nos locais mensalmente por um período de duas semanas, de março de 2006 a fevereiro de 2007.

As hortas foram caracterizadas segundo levantamento qualitativo das plantas cultivadas (nos dias de instalação e remoção das armadilhas) e das plantas infestantes no entorno das hortas em julho/06 e janeiro/07, épocas secas e úmidas, respectivamente. As similaridades entre as áreas em relação às plantas cultivadas e não cultivadas do entorno foram avaliadas por um dendrograma de similaridade, empregando-se a Análise Multivariada de Agrupamento pelo método das Médias não Ponderadas UPGMA (Unweighted Pair-Group Method Average). Os dendrogramas foram gerados pelo software Palaeontological Statistics / PAST (Hammer et al., 2001).

A influência dos fatores climáticos na abundância e riqueza de espécies de Eiphosoma foi avaliada pelo teste de correlação de Pearson. Os dados climáticos de precipitação $(\mathrm{mm})$, temperatura média e umidade relativa $(\%)$ foram fornecidos pela Embrapa Pecuária Sudeste, em São Carlos e pela Fazenda Rancho Rey, em Araraquara, ambas no Estado de São Paulo.

Foram utilizados cinco estimadores de riqueza: Jackknife 1 e 2, Chao 1 e 2 e Bootstrap. Os quatro primeiros baseiam-se na proporção de espécies raras em relação à riqueza total observada e empregam como medidas de raridade singletons e doubletons (espécies representadas por apenas um ou dois indivíduos respectivamente) ou uniques e duplicates (espécies que ocorrem em apenas uma ou duas amostras, respectivamente. O Bootstrap é calculado pela soma da riqueza observada com o inverso da proporção de amostras em que ocorre cada espécie (Colwell, 2005). As estimativas foram calculadas no programa EstimateS versão 8.0, com 100 aleatorizações (Colwell, 2005).

A diversidade e equitabilidade das espécies de Eiphosoma, em cada área estudada, foram calculadas 
pelos índices de diversidade de Shannon e equitabilidade de Pielou. As similaridades entre as áreas, em relação à fauna estudada, foram avaliadas por um dendrograma de similaridade, empregando-se a Análise Multivariada de Agrupamento pelo método das Médias não Ponderadas UPGMA (Unweighted Pair-Group Method Average). Os cálculos foram realizados no software Palaeontological Statistics / PAST (Hammer et al., 2001).

\section{Resultados e Discussão}

No total foram coletados 1.613 indivíduos pertencentes a 14 espécies de Eiphosoma. Das 17 espécies de Eiphosoma brasileiras conhecidas, foram identificadas 9 espécies. E. strontorium Gauld, 2000 e E. tantalium Gauld, 2000, registradas até o momento somente para Costa Rica (Gauld, 2000), são novas ocorrências para o país e 3 novas espécies estão sendo descritas.

Novos registros de ocorrência de espécies e a identificação de espécies ainda não descritas corroboram a teoria de que sistemas agrícolas mais diversificados são importantes ferramentas na conservação da biodiversidade alinhando os objetivos da agricultura com os da conservação natural (Landis et al., 2000).

A maior abundância de indivíduos foi obtida na Horta Oyafuso. As espécies E. dentator Fabricius, 1804, E. fluminense Costa Lima, 1953 e E. laphygmae Costa Lima, 1953 foram as mais abundantes nas três hortas estudadas. E. laphygmae foi mais abundante nas hortas Oyafuso e Prefeitura de São Carlos (50,1\% e 68,7\%, respectivamente); na Horta São Paulo, E. dentator foi mais abundante $(53,1 \%)$ (Tabela 1). Ambas as espécies estão relacionadas a importantes pragas agrícolas. E. dentator tem sido registrada como parasitoide de Diaphania hyalinata (Lepidoptera, Pyralidae), praga das curcubitáceas e E. laphygmae como parasitoide de espécies pertencentes ao gênero Spodoptera (Lepidoptera, Noctuidae), especialmente $S$. frugiperda, nocivas a diversas culturas (Gauld, 2000).

As dez espécies de Eiphosoma identificadas na Horta da Prefeitura também foram encontradas na Horta Oyafuso. Nas hortas São Paulo e Oyafuso, verificou-se a ocorrência de espécies exclusivas (duas e uma, respectivamente). O dendrograma de similaridade obtido para as espécies de Eiphosoma amostradas indicou maoior similaridade entre as hortas da Prefeitura de São Carlos e Horta Oyafuso (Figura 1).

A maior diversidade e equitabilidade de espécies foram observadas na Horta Oyafuso. Para as três hortas, os valores de equitabilidade obtidos foram muito baixos devido à dominância das três espécies citadas acima. As riquezas de espécies de Eiphosoma foram semelhantes nas três hortas, porém as estimativas de riqueza variaram muito entre seus estimadores (Tabela 2).

Os estimadores selecionados indicaram estimativas de riquezas de espécies próximas às reais

Tabela 1. Espécies de Eiphosoma (Hymenoptera, Ichneumonidae, Cremastinae) coletadas nas localidades estudadas. HO: Horta Oyafuso; HSP: Horta São Paulo; HPSC: Horta da Prefeitura de São Carlos.

\begin{tabular}{|c|c|c|c|c|}
\hline Espécies & $\mathrm{HO}$ & HSP & HPSC & Total \\
\hline E. dentator Fabricius, 1804 & 117 & 187 & 28 & 332 \\
\hline E. fluminense Costa Lima, 1953 & 180 & 46 & 46 & 272 \\
\hline E. laphygmae Costa Lima, 1953 & 464 & 89 & 231 & 784 \\
\hline E. lopesi Costa Lima, 1953 & 1 & 1 & 0 & 2 \\
\hline E. maculicoxa Enderlein, 1921 & 76 & 6 & 11 & 93 \\
\hline E. minense Costa Lima, 1953 & 0 & 2 & 0 & 2 \\
\hline E. ribeiroi Costa Lima, 1953 & 2 & 2 & 2 & 6 \\
\hline E. silvai Costa Lima, 1953 & 0 & 1 & 0 & 1 \\
\hline E. strontorium Gauld, 2000 & 7 & 3 & 3 & 13 \\
\hline E. tantalium Gauld, 2000 & 1 & 2 & 1 & 4 \\
\hline E. travassosi Costa Lima, 1953 & 62 & 11 & 10 & 83 \\
\hline Eiphosoma sp 1 & 13 & 2 & 1 & 16 \\
\hline Eiphosoma sp 2 & 1 & 0 & 0 & 1 \\
\hline Eiphosoma sp 3 & 1 & 0 & 3 & 4 \\
\hline
\end{tabular}




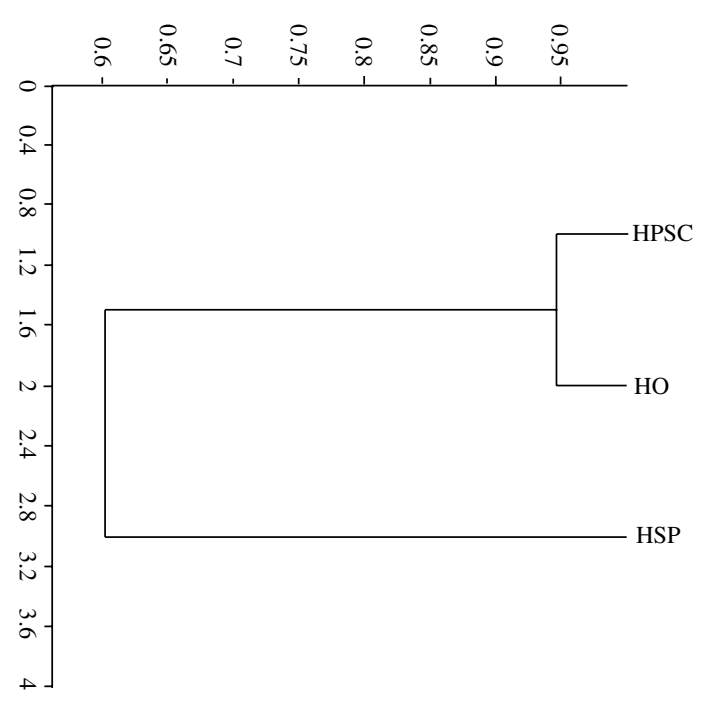

Figura 1: Dendrograma de similaridade entre os locais estudados com base na fauna de Eiphosoma (Hymenoptera, Ichneumonidae, Cremastinae) amostrada. (Coeficiente de similaridade por Morisita: $r=0,96)$. HO: Horta Oyafuso; HSP: Horta São Paulo; HPSC: Horta da Prefeitura de São Carlos.

observadas. Na Horta da Prefeitura, o Chao 1 indicou que praticamente todas as espécies foram coletadas e, segundo os estimadores Bootstrap e Jackniffe 1, uma a duas espécies poderiam ter sido coletadas. Na Horta São Paulo, cerca de 3 espécies poderiam ter sido coletadas pelo estimador Jackniffe 1, assim como na Horta Oyafuso.

Foram observados os maiores valores de raridade nas hortas Oyafuso e Horta São Paulo, na maioria das vezes, envolvendo as mesmas espécies. A Horta Oyafuso apresentou quatro singletons e uniques: $E$. tantalium Gauld, 2000, E. lopesi Costa Lima, 1953, Eiphosoma sp 2 e Eiphosoma sp 3. A Horta São Paulo também apresentou quatro uniques: $E$. ribeiroi Costa Lima, 1953, E. silvai Costa Lima, 1953, E. lopesi Costa Lima, 1953 e Eiphosoma sp 1. Os maiores valores de doubletons e duplicates foram obtidos na Horta São Paulo: três doubletons (E. minense Costa Lima, 1953, E. ribeiroi Costa Lima, 1953 e E. tantalium Gauld, 2000) e três duplicates (E. maculicoxa Enderlein, 1921, E. tantalium Gauld, 2000 e E. minense Costa Lima, 1953) (Tabela 2).

As variáveis abióticas estudadas tiveram pouca ou nenhuma influência sobre a fauna de Eiphosoma. As correlações mais significativas foram obtidas na Horta São Paulo, provavelmente, resultado da manutenção das plantas cultivadas por um período mais extenso e da menor intensidade de manejo humano (Tabela 3).

A Horta Oyafuso apresentou um maior número de espécies de plantas não cultivadas e infestantes (56), seguidas pela Horta da Prefeitura (52) e Horta São Paulo (33). Em relação às plantas cultivadas, a Horta da Prefeitura apresentou maior número de espécies de plantas cultivadas (40), seguida pela Horta São Paulo e Horta Oyafuso (30 e 24 espécies, respectivamente) (Figuras 2 e 3 ).

As plantas cultivadas e infestantes, o manejo e tamanho das hortas tiveram maior influência que as variáveis climáticas na fauna de Eiphosoma. Isto era esperado, uma vez que os sistemas agrícolas possuem maior dinâmica de manejo e de perturbação do que os ambientes naturais.

Entretanto, esperava-se que na Horta São Paulo, devido à menor área, menor densidade de cultivos e de vegetação infestante, a fauna de Eiphosoma fosse menos abundante, rica e diversa do que nas demais hortas estudadas.

A diversidade vegetal dentro e no entorno dos agroecossistemas pode favorecer positivamente as populações de inimigos naturais, oferecendo abrigo, alimento, sítios de acasalamento e hospedeiros

Tabela 2. Descritores da fauna de Eiphosoma (Hymenoptera, Ichneumonidae, Cremastinae) nas localidades estudadas: abundância, riqueza de espécies observada, estimadores de riqueza, diversidade e equitabilidade. HO: Horta Oyafuso; HSP: Horta São Paulo; HPSC: Horta da Prefeitura de São Carlos.

\begin{tabular}{lccc}
\hline & HO & HSP & HPSC \\
\hline Abundância & 925 & 352 & 336 \\
Riqueza observada & 12 & 12 & 10 \\
Chao 1 & 15,2 & 12,2 & 10,5 \\
Chao 2 & 20,4 & 15,37 & 11,83 \\
Jackniffe 1 & 15,67 & 15,67 & 11,83 \\
Jackniffe 2 & 18,24 & 17,16 & 12,71 \\
Bootstrap & 13,52 & 13,78 & 10,96 \\
Singletons & 4 & 2 & 2 \\
Doubletons & 1 & 3 & 1 \\
Uniques & 4 & 4 & 2 \\
Duplicates & 1 & 3 & 2 \\
Diversidade de Shannon & 1,452 & 1,319 & 1,103 \\
Equitabilidade & 0,5844 & 0,5307 & 0,479 \\
\hline
\end{tabular}


Tabela 3. Valores de correlação entre abundância, riqueza de espécies de Eiphosoma (Hymenoptera, Ichneumonidae, Cremastinae) e variáveis climáticas. (HO: Horta Oyafuso; HSP: Horta São Paulo; HSPC: Horta Prefeitura de São Carlos; A: abundância de indivíduos; S: riqueza de espécies).

\begin{tabular}{lcclrc}
\hline Variáveis & $\mathrm{A}$ & $\mathrm{S}$ & Variáveis & $\mathrm{A}$ & $\mathrm{S}$ \\
\hline Temperatura HO & 0.28285 & -0.37179 & Precipitação HSP & $0.65428^{*}$ & 0.50906 \\
Umidade HO & 0.01096 & $-0.69537^{*}$ & Temperatura HPSC & -0.27395 & -0.49717 \\
Precipitação HO & 0.35914 & -0.29926 & Umidade HPSC & 0.01914 & -0.30424 \\
Temperatura HSP & $0.6568^{*}$ & $0.63138^{*}$ & Precipitação HPSC & -0.50779 & $-0.57966^{*}$ \\
Umidade HSP & 0.49709 & $0.64783^{*}$ & & & \\
\hline
\end{tabular}

* Correlação significativa $(\mathrm{p}<0.05)$.

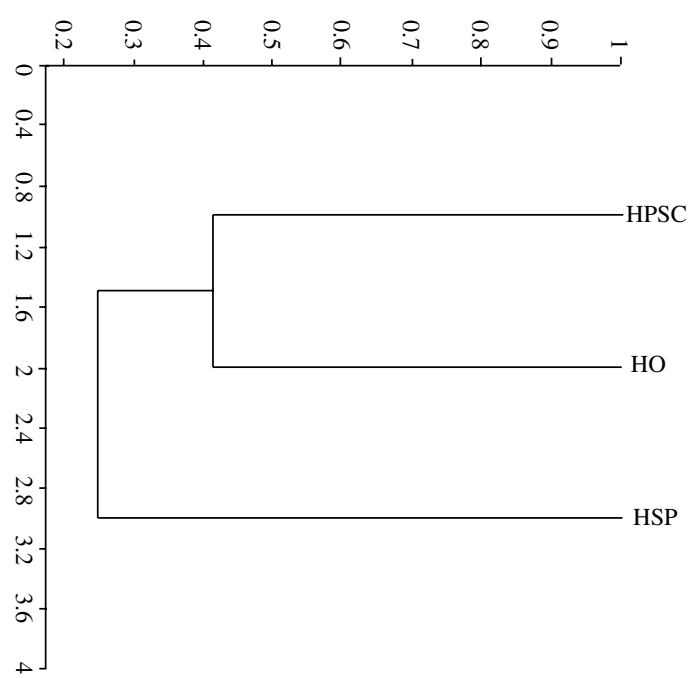

Figura 2. Dendrograma de similaridade entre os locais estudados com base na composição de plantas infestantes. (Coeficiente de similaridade por Jaccard). HO: Horta Oyafuso; HSP: Horta São Paulo; HPSC: Horta da Prefeitura de São Carlos.

alternativos (Chay-Hernandez, et al., 2006, Landis et al., 2000). Na Horta da Prefeitura, os benefícios gerados pela diversidade das plantas podem ter sido reduzidos pela alta intensidade de manejo.

\section{Conclusões}

A fauna de Eiphosoma é rica e abundante em hortas orgânicas. Sua composição, abundância e riqueza de espécies pode ser afetada pela intensidade e forma de manejo, porém a tendência observada no presente estudo poderia ser melhor comprovada

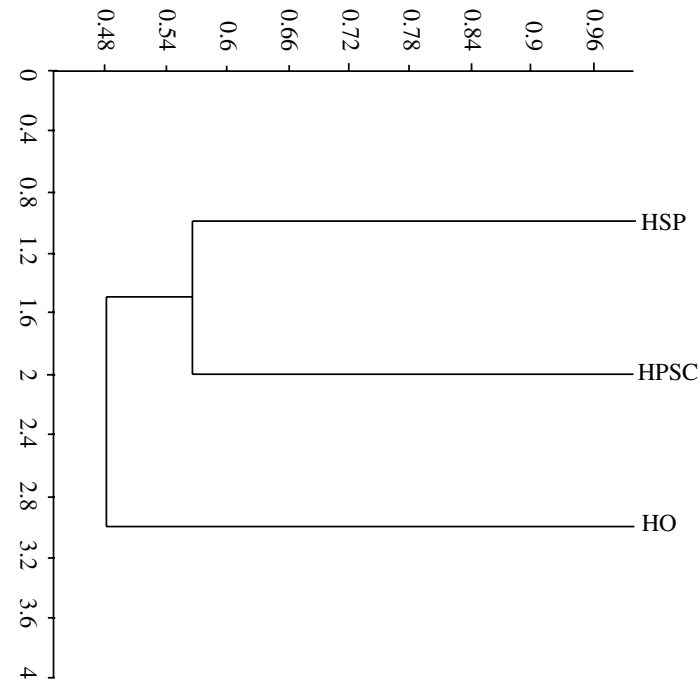

Figura 3. Dendrograma de similaridade entre os locais estudados com base na composição de plantas cultivadas. (Coeficiente de similaridade por Jaccard). HO: Horta Oyafuso; HSP: Horta São Paulo; HPSC: Horta da Prefeitura de São Carlos.

com a realização de estudos contendo um maior número de propriedades.

\section{Agradecimentos}

À Sr(a) Leoni e Magda Yamada, Sr. Marcelo Oyafuso e à Prefeitura de São Carlos e aos seus funcionários, por permitirem o acesso às áreas de estudo; à FAPESP (Fundação de Amparo à Pesquisa do Estado de São Paulo) e CNPq (Conselho Nacional de Desenvolvimento Científico e Tecnológico) pelo suporte financeiro. 


\section{Referências Bibliográficas}

Altieri, M.A.

1994 Biodiversity and pest management in agroecosystems. New York, Food Products Press. 185 p.

Altieri, M. A.; Silva, E. N.; Nicholls, C. I.

2003 O papel da biodiversidade no manejo de pragas. Ribeirão Preto: Holos, pp. 52-71.

Chay-Hernandez, D. A.; Delfin-Gonzalez, H.; Parra-Tabla, V. 2006 Ichneumonoidae (Hymenoptera) Community Diversity in an Agricultural Environment in the State of Yucatan, Mexico. Environmental Entomology, v. 35, pp. 1286-1297. Colwell, R. K.

2005 Estimates: Statistical estimation of species richness and shared species from samples. Version 8.0. 2005. User's Guide and application. Disponível em: http://purl.oclc.org/ estimates. Acesso em 10 de agosto de 2008.

Fernández-Triana, J. L.; Ravelo, H. G.

2007 A taxonomic review of Cuban Eiphosoma Cresson (Hymenoptera: Ichneumonidae) with biogeographical notes. Zootaxa, pp. 49-61.
Gauld, I. D.

2000 The Ichneumonidae of Costa Rica. 3. Memoirs of American Entomological Institute, v. 63, pp. 1-453.

Hammer, O.; Harper, D.A.T. \& Ryan, P.D.

2001 Paleontological statistics. Version 1.34. Disponível em: http// www.folk.uio.no/ohammer/past. Acesso em 10 de agosto de 2008.

Landis, D. A.; Wratten, S. T.; Gurr, G. M.

2000 Habitat management to conserve natural enemies of arthropod pests in agriculture. Annual Review of Entomology, v. 45 , pp. $175-201$.

Wyss, E.; Pfiffner, L.

2006 Biodiversity in organic horticulture-an indicator for sustainability and a tool for pest management. In: XXVII International Horticultural Congress. 2006. Acta Horticulturae 767. 